\title{
BLOCK INTERSECTIONS \\ IN BALANCED INCOMPLETE BLOCK DESIGNS
}

\author{
R. G. Stanton and D. A. Sprott
}

(received January 27, 1964)

1. Introduction. One of the most interesting of the smaller $B I B D^{\prime} s$ is the system $(8,14,7,4,3)$, where we write the parameters in the standard order $v, b, r, k, \lambda$. One representation of a design with the se parameters is 1248,3567; 2358,$1467 ; 3468,1257 ; 4578,1236 ; 5618,2347 ; 6728,1345$;

7138,2456 . This particular design has the feature that every block $B$ is paired with a complementary block $B^{\prime}$ consisting of all varieties not lying in $B$. Thus $B \cap B^{\prime}=0$. If we seek to generalize this type of design, we obtain

THEOREM 1. If a design contains one pair of complementary blocks, then it must have parameters

$$
2 x+2, t(4 x+2), t(2 x+1), x+1, t x
$$

Proof. Let the number of plots in a block be $k=x+1$. Since all varieties occur in a pair of complementary blocks $B$ and $B^{\prime}$, it follows that $\nabla=2(x+1)$. Also, the basic $B I B D$ relations give

$$
\lambda(2 x+1)=r x, 2 r=b .
$$

Since $\mathbf{x}$ is relatively prime to $2 x+1, x$ must divide $\lambda$, say $\lambda=\mathrm{tx}$. The theorem now follows.

It will be convenient to refer to the designs with parameters as specified in Theorem 1 as designs $\mathrm{H}_{2}(t, x)$; a generalization will be given later. It should of course be pointed out that,

Canad. Math. Bull. vol. 7, no. 3, July 1964. 
while we have shown that every design which splits into pairs of complementary blocks is automatically a design $\mathrm{H}_{2}(t, x)$, it does not follow that a design $\mathrm{H}_{2}(t, x)$ necessarily possesses the splitting property which we have discussed.

The simplest designs $H_{2}(t, x)$ are the designs $H_{2}(1, x)=H_{2}(x)$; we shall obtain certain results about the se designs, and extend one of the results to designs in general. However, designs which have a factor in common among $b, r$, and $\lambda$, need not be ignored as implied by Parker [3]. The useful design made up of all selections of triplets from 7 varieties has parameters $(7,35,15,3,5)$, yet no blocks are repeated; on the other hand, one can get a different design with these parameters by repeating the Fano design $(7,7,3,3,1)$ a total of five times. Also, the design $(16,8 \lambda, 3 \lambda, 6, \lambda)$ exists for all $\lambda>1$, but not for $\lambda=1$.

2. Block Intersection Properties. Parker [3] showed that for $x$ odd it was not possible for two blocks of a design $\mathrm{H}_{2}(\mathrm{x})$ to be identical; Seiden [4] extended this result to all $\mathrm{x}$ by using the theory of orthogonal arrays. In Theorem 2, we shall deduce this result by using a technique which is originally due to Fisher [2] and which has also been used by Bose [1].

THEOREM 2. In a design $\mathrm{H}_{2}(\mathrm{x})$, it is impossible to have two identical blocks.

Proof. Let $B_{1}$ be a specific block and let $x_{i}=x_{1 i}$ be the number of elements in $B_{1} \cap B_{i}$, where $i$ ranges from 2 to b. It immediately follows that, in general,

$$
\begin{aligned}
\bar{x} & =\Sigma x_{i} /(b-1)=k(r-1) /(b-1), \\
\Sigma\left(x_{i}-\bar{x}\right)^{2} & =k(\lambda k-k-\lambda+r)-k^{2}(r-1)^{2} /(b-1)
\end{aligned}
$$

For the designs $H_{2}(x)$, we find 


$$
\bar{x}=2 x(x+1) /(4 x+1)
$$

$$
\Sigma\left(x_{i}-\bar{x}\right)^{2}=(x+1)^{2} x /(4 x+1)
$$

If there is another block $B_{j}$ identical with $B_{1}$, then $x_{j}=k$ and

$$
\left(x_{j}-\bar{x}\right)^{2}=(x+1)^{2}(2 x+1)^{2} /(4 x+1)^{2} \text {. }
$$

Since $\Sigma\left(x_{i}-\bar{x}\right)^{2}-\left(x_{j}-\bar{x}\right)^{2} \geq 0$,

we arive at the contradiction

$$
(x+1)^{2}(-1-3 x) /(4 x+1)^{2} \geq 0
$$

It follows that there cannot be a block $B_{j}$ identical with $B_{1}$. Since $B_{1}$ was arbitrary, the theorem follows.

The same method allows us to discuss the possibility of complementary blocks in $\mathrm{H}_{2}(\mathrm{x})$.

THEOREM 3. If $x_{2}=0$, then $x$ must be odd. Furthermore, all other values of $x_{i}$ must be equal to $\frac{1}{2}(x+1)$. Proof. The first part of this theorem was proved by
Parker [3], using incidence matrices. We note that if a block, say $B_{2}$, is complementary to $B_{1}$, then $x_{2}=0$. Consider the $b-2$ variates $x_{3}, x_{4}, \ldots, x_{b}$. Then

$$
\begin{aligned}
& \sum_{i=3}^{b} x_{i}=\sum_{i=2}^{b} x_{i}=k(r-1)=2 x(x+1), \bar{x}=\frac{1}{2}(x+1) ; \\
& \sum_{i=3}^{b} x_{i}^{2}=(x+1)^{2} x .
\end{aligned}
$$


Clearly $\sum_{i=3}^{b}\left(x_{i}-\bar{x}\right)^{2}=(x+1)^{2} x-4 x\left[\frac{1}{2}(x+1)\right]^{2}=0$.

Thus $x_{i}=\bar{x}=\frac{1}{2}(x+1)$ for $i \geq 3$. Since $x_{i}$ is an integer, we see that $x$ must be odd. Furthermore, if $x$ is odd and there are two complementary blocks, then the se two blocks intersect any other block in the same number of varieties, namely, $\frac{1}{2}(x+1)$.

3. A Generalization of the Fisher Inequality. Fisher's inequality $b \geq v$ was proved in [2]; we use the method of Section 2 to prove

THEOREM 4. If a BIBD contains $\alpha>0$ blocks other than $B_{1}$ which are identical with a specified block $B_{1}$, then $\mathrm{b} \geq(\alpha+1) \mathrm{v}-(\alpha-1)$.

Proof. Define $T$ by the equation

$$
T=\Sigma\left(x_{i}-\bar{x}\right)^{2}=k(k \lambda-k-\lambda+r)-k^{2}(r-1)^{2} /(b-1) .
$$

Using the basic relation

$$
r-\lambda=r k-\lambda v,
$$

we write

$$
\begin{aligned}
T & =k(k \lambda-k+r k-\lambda v)-k^{2}(r-1)^{2} /(b-1) \\
& =k \lambda(k-v)+k^{2}(r-1)-k^{2}(r-1)^{2} /(b-1) \\
& =k \lambda(k-v)+k^{2}(r-1)(b-r) /(b-1) .
\end{aligned}
$$

Now the basic relation $b / r=v / k$ can be written as

$$
(b-r) / r=(v-k) / k \text {, }
$$

so we obtain

$$
T=k^{2}(r-1)(b-r) /(b-1)-k^{2} \lambda(b-r) / r ;
$$


but the contribution from the blocks identical with $B_{1}$ is

$$
\alpha(k-\bar{x})^{2}=\alpha k^{2}(b-r)^{2} /(b-1)^{2}
$$

and this cannot exceed $T$. We thus find

$$
\alpha k^{2}(b-r) /(b-1)^{2} \leq k^{2}(r-1) /(b-1)-k^{2} \lambda / r .
$$

This relation may be written as

$$
\alpha(b-r) /(b-1) \leq\left(r^{2}-b \lambda-r+\lambda\right) / r .
$$

Now we may use (3.1) to write

$$
\begin{aligned}
b-r & =(b k-r k) / k=(r v-r k) / k=(r v+\lambda-r-\lambda v) / k \\
& =(r-\lambda)(v-1) / k .
\end{aligned}
$$

Also, by another use of (3.1),

$$
r^{2}-b \lambda=\left(k r^{2}-b k \lambda\right) / k=\left(k r^{2}-r v \lambda\right) / k=r(r-\lambda) / k .
$$

Our inequality may then be written

$$
\alpha(r-\lambda)(v-1) / k(b-1) \leq(r-\lambda) / k-(r-\lambda) / r ;
$$

since $r-\lambda>0$, we find

$$
\alpha b k(v-1) / v \leq(b-1)(r-k)=(b-1)(b k / v-k) \text {, }
$$

$$
b \alpha(v-1) \leq(b-1)(b-v)
$$

If we put $\alpha=0$ in (3.3), we immediately obtain Fisher's result $b \geq v$. Assuming $\alpha \neq 0$, we can write (3.3) as

$$
b^{2}-b v+v \geq a b(v-1)+b
$$

and so obtain

$$
b^{2}+v \geq b v(\alpha+1)-b(\alpha-1)
$$




$$
b \geq v(\alpha+1)-(\alpha-1)-\frac{v}{b}
$$

In this inequality $\mathrm{b}=\mathrm{v}$ is not possible. Thus $\mathrm{v} / \mathrm{b}<1$; but $b$ is an integer, and so

$$
\mathrm{b} \geq(\alpha+1) \mathrm{v}-(\alpha-1)
$$

This establishes the theorem.

We note that $\alpha=1$ implies that $b \geq 2 v$; consequently, the condition that there be no repeated block leads to the restriction $b<2 v$. We then obtain

THEOREM 5. For a given value of $v$, the design having largest $b$ for which there is no possibility of a repeated block is just the design $\mathrm{H}_{2}(\mathrm{x})$.

Proof. If there is to be no repeated block, the restriction $\mathrm{b}<2 \mathrm{v}$ forces us to try $\mathrm{b}=2 \mathrm{v}-1$. This value is impossible, since the equation

$$
(2 v-1) k=r v
$$

leads to the contradiction that $v$ must divide $k$. Thus we must try $b=2 v-2$. Then we obtain

$$
(2 v-2) k=r v, \lambda(v-1)=r(k-1) .
$$

It follows from the first of the se equations that $v=2 k, r=v-1$; from the second we then obtain $\lambda=k-1$. Our design is then

$$
(2 k, 4 k-2,2 k-1, k, k-1)=H_{2}(k-1)
$$

4. The Family $H_{n}(x)$. If we seek to generalize the results of Section 2, we obtain

THEOREM 6. If a design contains a set $S$ of $n$ disjoint blocks forming a complete replication, then $r \geq k+\lambda$.

Proof. Let the blocks in $S$ be $B_{1}, \ldots, B_{n}$. Then 
$v=n k$ and $b=n r$, so there are $n(r-1)$ blocks outside $S$. Also, let $x_{j}$ be the number of varieties in $B_{1} \cap B_{j}$, where $j=n+1, \ldots, b$. We find, as usual,

$$
\begin{aligned}
& \Sigma x_{j}=k(r-1), \sum x_{j}^{2}=k(\lambda k-\lambda-k+r) . \\
& \bar{x}=\frac{k(r-1)}{n(r-1)}=\frac{k}{n} .
\end{aligned}
$$

Then

$$
\Sigma\left(x_{j}-\bar{x}\right)^{2}=k(\lambda k-\lambda-k+r)-\frac{k^{2}(r-1)}{n} \geq 0 .
$$

So

$$
\begin{aligned}
& n k(\lambda k-\lambda-k+r)-k^{2}(r-1) \geq 0, \\
& v(\lambda k-\lambda-k+r)-k^{2}(r-1) \geq 0, \\
& k(\lambda v-r k)+v(r-\lambda)-k v+k^{2} \geq 0, \\
& -k(r-\lambda)+v(r-\lambda)-k(v-k) \geq 0 .
\end{aligned}
$$

Divide by $v-k>0$ to give the result $r-\lambda-k \geq 0$, that is $\mathbf{I} \geq \mathrm{k}+\lambda$.

It is well known (see for example Stanton [5]) that the condition $r \geq k+\lambda$ is equivalent to the condition $v \geq b+r-1$ given by Bose [1] for a resolvable design; However, we see here that this condition follows from the existence of a single set $S$ (in a resolvable design, there are $\mathbf{r}$ sets of blocks, each forming a complete replication).

Bose [1] showed that if one had an affine resolvable design, that is, a resolvable design in which blocks from different replications have the same number of elements in common, then $b=v+r-1$; conversely, if $b=v+r-1$, the design is affine resolvable. This idea generalizes to give

THEOREM 7. If a design contains a set $S$ of $n$ disjoint blocks forming a complete replication, and if $\mathbf{r}=k+\lambda$, then each block of $S$ has the same number of elements in common- 
with blocks outside $S$; moreover, $v$ divides $k^{2}$.

Proof. If $r=k+\lambda$ in Theorem 6, then $\Sigma\left(x_{j}-\bar{x}\right)^{2}=0$, that is,

$$
x_{j}=\bar{x}=\frac{k}{n}=\frac{k^{2}}{n k}=\frac{k^{2}}{v} \text {. }
$$

This result shows that $x_{j}$ is constant; furthermore, since $x_{j}$ is an integer, $v$ must divide $k^{2}$.

We can now use the results of Theorems 6 and 7 to obtain a series $H_{n}(x)$ generalizing the results of Section 2 .

THEOREM 8. Let a design contain a set $S$ of $n$ disjoint blocks forming a complete replication; also, let $r=k+\lambda$. Then the design, which we shall call $H_{n}(x)$, has parameters

$$
n(n x-x+1), n(n x+1), n x+1, n x-x+1, x
$$

Proof. We have

$$
\begin{aligned}
& v=n k, \quad b=n r ; \\
& \lambda(v-1)=r(k-1), \\
& r=k+\lambda .
\end{aligned}
$$

Then

$$
\begin{aligned}
& \lambda(n k-1)=r(k-1)=(k+\lambda)(k-1), \\
& \lambda n=k+\lambda-1=r-1 .
\end{aligned}
$$

So $n$ divides $r-1$, and we may thus set $r-1=n x$; the theorem follows.

COROLLARY $1 . \mathrm{n}$ is a factor of $\mathrm{x}-1$.

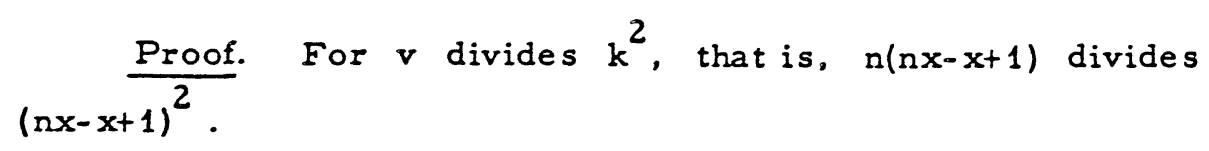


COROLLARY 2. Each block of $S$ in the design $H_{n}(x)$ intersects all blocks outside $S$ in $x-(x-1) / n$ varieties.

COROLLARY 3. If we drop the assumption $r=k+\lambda$ in Theorem 8, we obtain parameters

$$
\begin{aligned}
& v=n\left[1+\frac{x(n-1)}{y}\right], b=n(y+n x), \\
& r=y+n x, k=1+\frac{x(n-1)}{y}, \lambda=x,
\end{aligned}
$$

where $r-\lambda=k y$.

Proof. The relations $v=n k, b=n r, \lambda(v-1)=r(k-1)$, at once give $r-\lambda=k(r-\lambda n)$. So we may set $r-\lambda=k y$. We then obtain $y=r-\lambda n$, whence $k=\frac{n x-x+y}{y}$. The corollary follows. Evidently it is necessary that $y$ divide $x(n-1)$; the theorem corresponds to the case $y=1$. We can also use Theorem 4 to prove

THEOREM 9. The general family $\mathrm{H}_{n}(\mathrm{x})$ cannot have repeated blocks.

Proof. Let $\alpha(\alpha \geq 1)$ be the number of blocks, other
than $B_{1}$ itself, identical with $B_{1}$. Then

$$
\mathrm{b} \geq(\alpha+1) v-(\alpha-1)
$$

For $H_{n}(x)$, we find

$$
\begin{aligned}
& n(n x+1) \geq(\alpha+1) n(n x-x+1)-\alpha+1, \\
& n^{2} x-n(n-1)(\alpha+1) x \geq n(\alpha+1)-\alpha+1-n, \\
& n x(-n \alpha+\alpha+1) \geq \alpha(n-1)+1 .
\end{aligned}
$$

Now $\mathrm{n}$ and $\mathrm{x}$ are fixed and positive; $\alpha$ must be chosen so that $\alpha+1-n \alpha>0$, that is, $\alpha(1-n)+1>0$. This cannot occur since $n \geq 2, \alpha \geq 1$, We have thus established the theorem. 
5. Conclusion. Interesting questions arise concerning the designs $\mathrm{H}_{2}(t, x)$ with $t>1$, non-isomorphic designs $\mathrm{H}_{2}(x)$, the existence of designs $\mathrm{H}_{2}(x)$ with prescribed block intersection numbers satisfying the relation (2.1), discussion of other series of designs. Studies along the se lines are under way.

\section{REFERENCES}

1. R. C. Bose, A note on the resolvability of balanced incomplete block designs, Sankhya 6 (1942), 105-110.

2. R.A. Fisher, An examination of different possible solutions in incomplete blocks, Ann. Eugenics 10 (1940), $52-75$.

3. E. T. Parker, Remarks on balanced incomplete block designs, Proc. Amer. Math. Soc. 14 (1963), 729-730.

4. E. Seiden, A supplement to Parker's "Remarks on balanced incomplete block designs", Proc. Amer. Math. Soc. 14 (1963), 731-732.

5. R. G. Stanton, A Note on BIBDs, Ann. Math. Stat. 28 (1957), 1054-1055.

University of Waterloo 